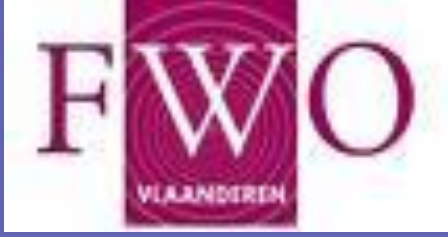

\title{
Association between bone mineral density and hearing loss in Osteogenesis Imperfecta
}

\section{F Swinnen ${ }^{1}$ E De Leenheer ${ }^{1}$ S Goemaere ${ }^{2}$ P Coucke $^{3}$ C Cremers ${ }^{4}$ I Dhooge ${ }^{1}$}

(1) Departement of Otorhinolaryngology, Ghent University (Hospital)

(2) Department of Endorinology \& Rheumatology, Unit Osteporosis \& Metabolic Bone Diseases, Ghent University Hospital

(3) Center for Medical Genetics, Ghent University Hospital

(4) Department of Otorhinolaryngology, Radboud University Nijmegen Medical Center 


\section{Osteogenesis Imperfecta (OI) - Hearing loss}

a $50 \%$ of Ol patients

Ol types I, III, IV

Mild to profound hearing loss, progressive

I Intrafamilial variability

- Hearing loss type:

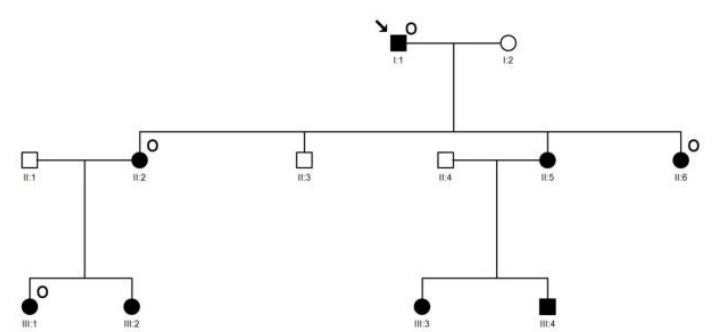

Conductive hearing loss

Pure high-frequency sensorineural hearing

loss

\section{Mixed hearing loss}

Pure sensorineural hearing loss 


\section{Ol - Hearing loss (2)}

\section{Mixed hearing loss}

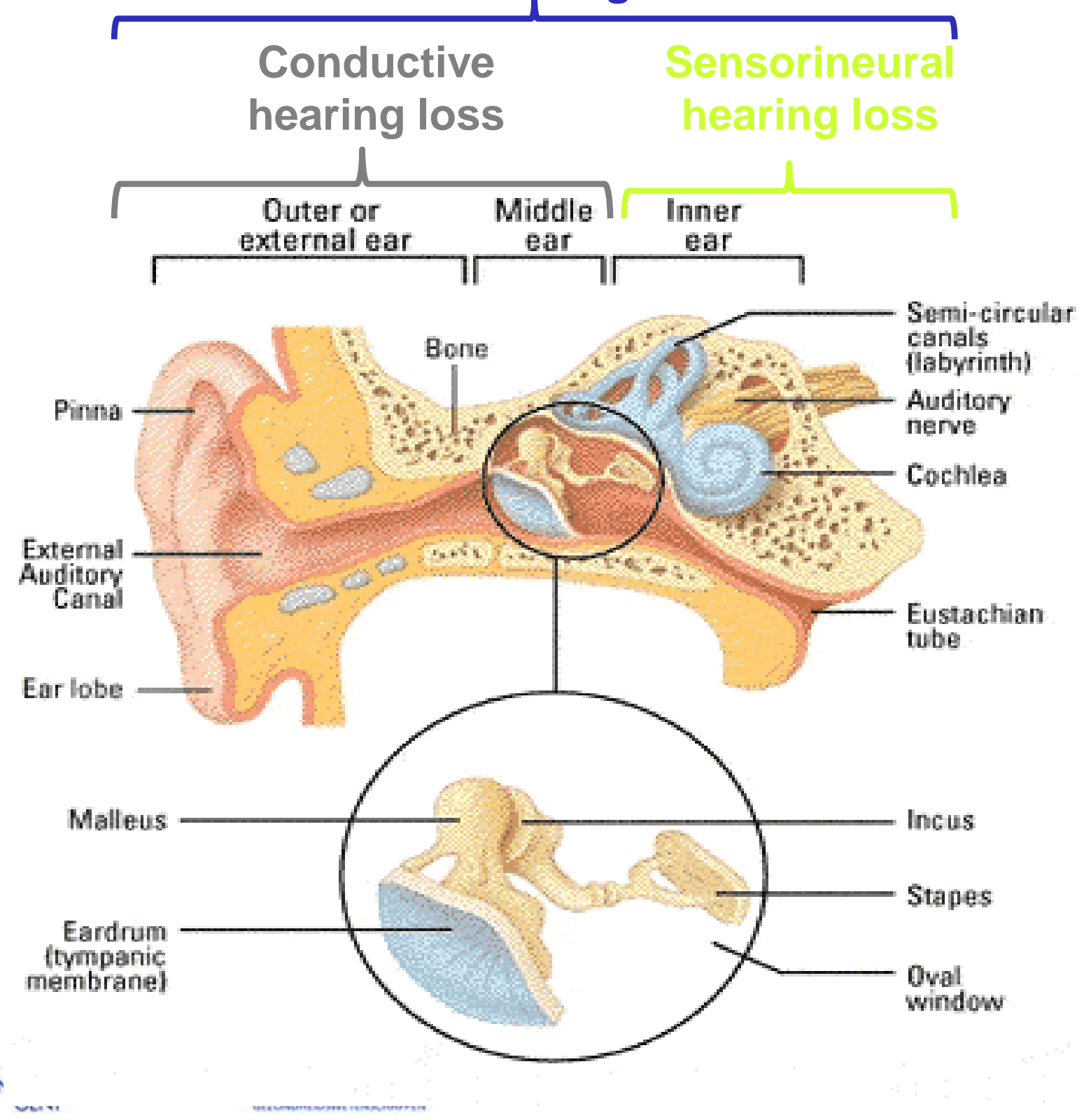




\section{OI - Hearing loss (3)}

\section{Conductive - Mixed}

- Otosclerosis-like lesions: stapes footplate fixation (and pericochlear lesions)

- Ossicular discontinuity (fractured/atrophic ossicles)

\section{Pure sensorineural loss}

- Cochlear hair cell atrophy

- Atrophy stria vascularis

․ Perilymphe hemorrhage 
Computed tomography temporal bones

Bilaterally severely progressed mixed hearing loss in a 67-year old OI-patient: severe pericochlear demineralization of bone
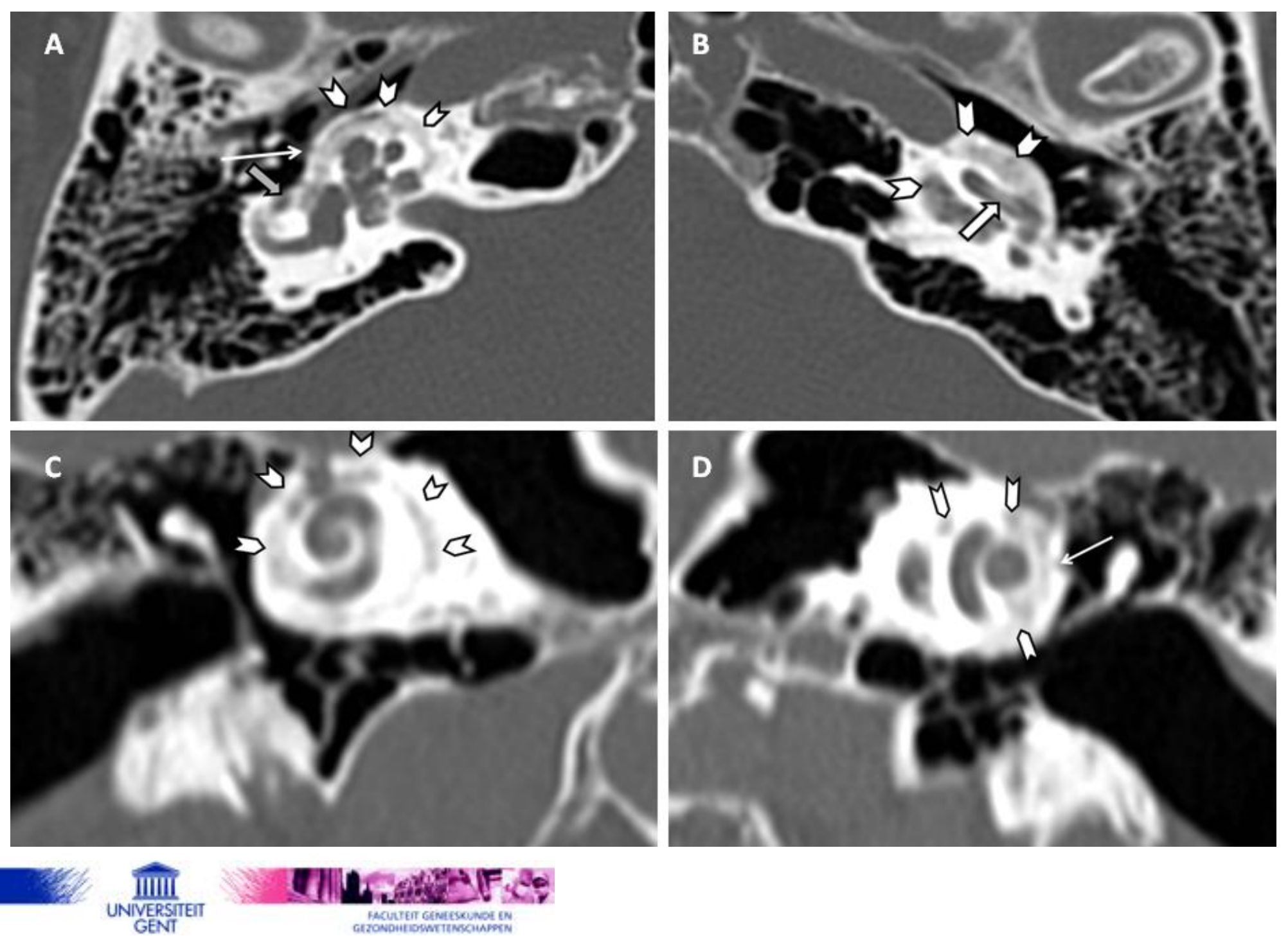

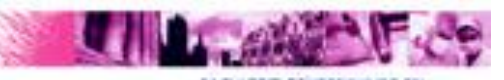

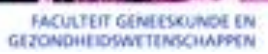




\section{Research aim}

$\square$ Relationship between occurrence/type of hearing loss and generalized bone disease?

Heterogeneity of hearing loss explained by variability in bone characteristics? 


\section{Patients and materials}

口 56 adult OI patients ( $\mathrm{F}: 34 \mathrm{M}: 22$ ) with identified mutation in COL1A1 or COL1A2

$\square$ Mean age: 43 y. (SD 13.7)

$\square$ Bisphosphonates administration excluded

- Audiological evaluation

$\square$ Pure-tone audiometry

$\square$ Admittance measurements

$\square$ Stapedius reflex measurements

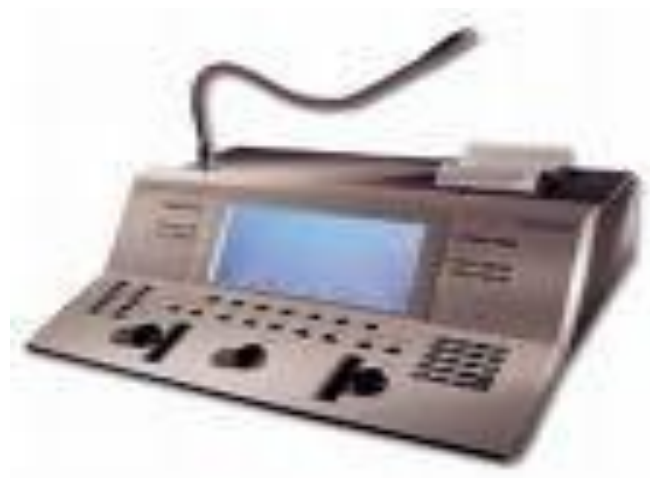

$\square$ Bone mineral density (BMD) measurements 


\section{Bone mineral density measurements}

$\square$ Dual X-ray absorptiometry (DXA): areal BMD (aBMD)

- Lumbar spine trabecular bone aBMD

- Whole body cortical bone aBMD
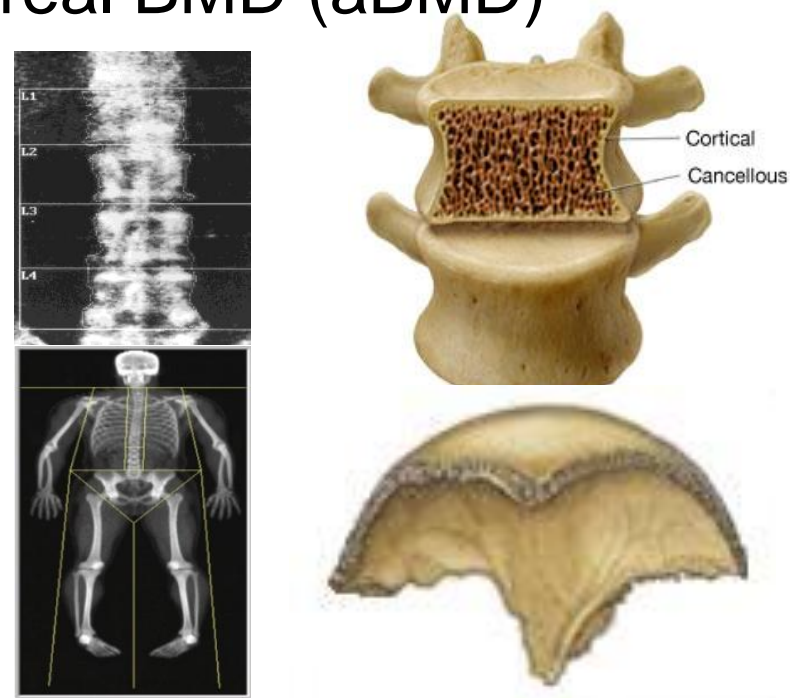

- Peripheral quantitative computed tomography ( $\mathrm{pQCT}$ ): volumetric $\mathrm{BMD}$ (vBMD)

- Radial metaphysis (4\%) trabecular bone vBMD

- Radial diaphysis (66\%) cortical bone vBMD

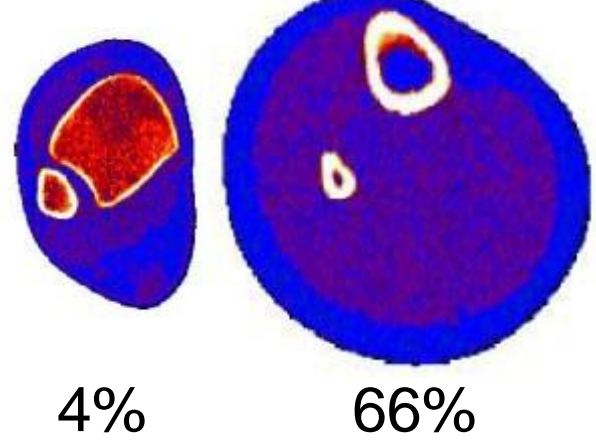
bone geometry parameters: cortical thickness, periosteal circumference, endosteal circumference 
Audiological phenotype

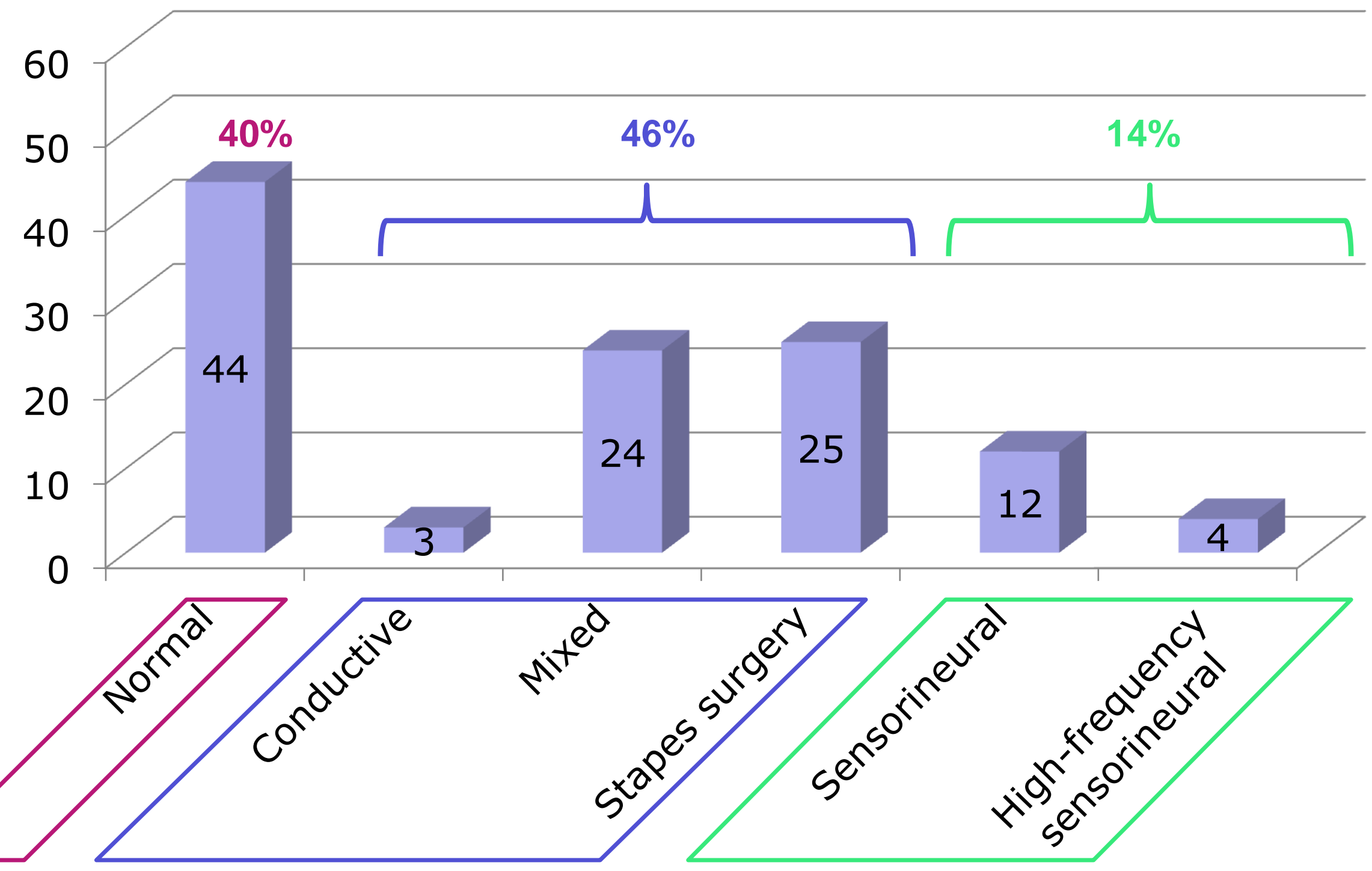

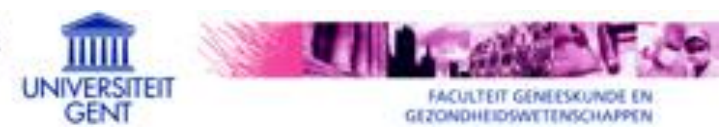




\section{Hearing loss as a function of Ol type and genotype in 56 Ol patients}

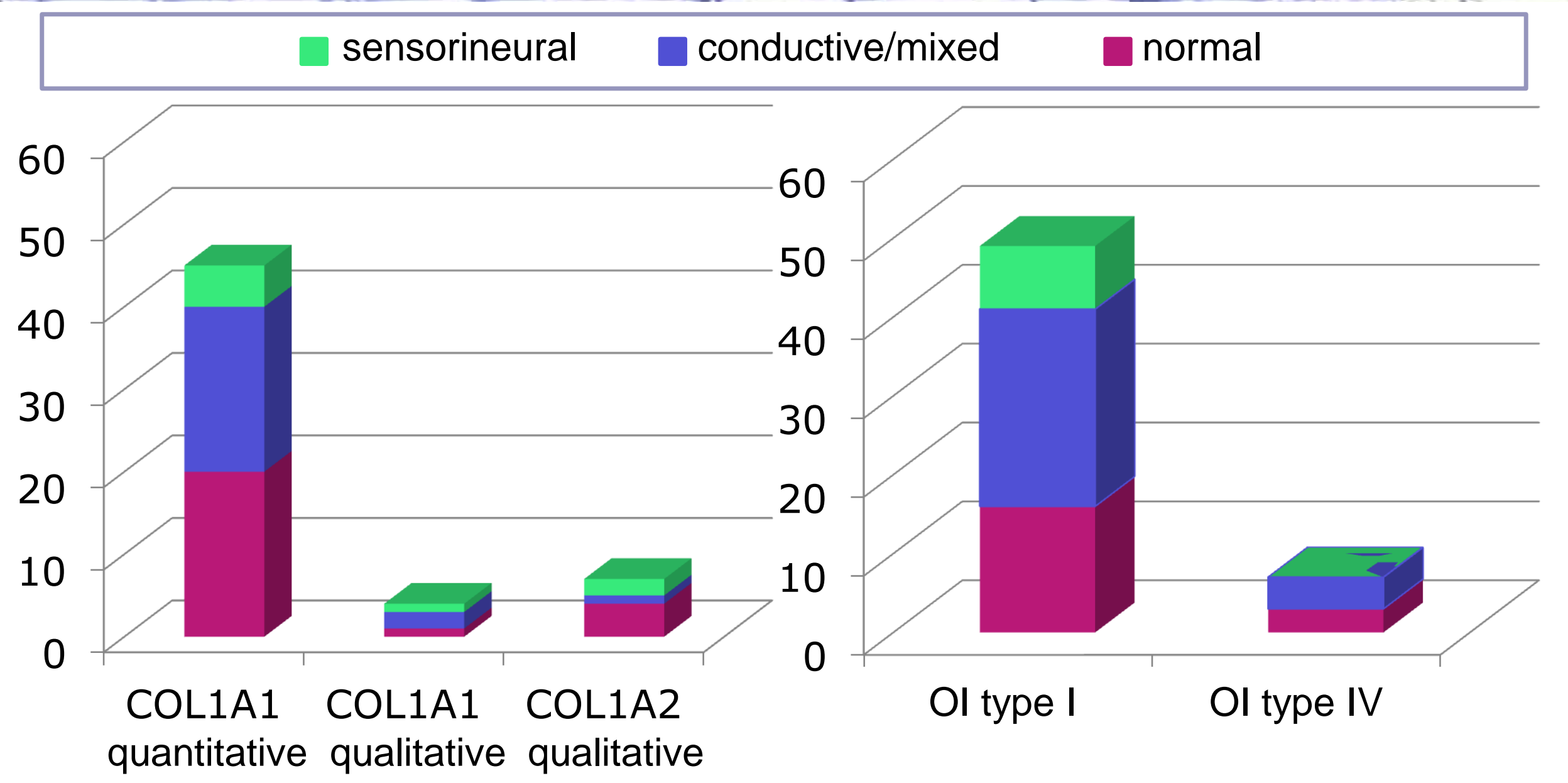

No association between hearing loss and mutated gene, type I collagen defect or Ol type 


\section{Hearing loss as a function of aBMD (DXA)}

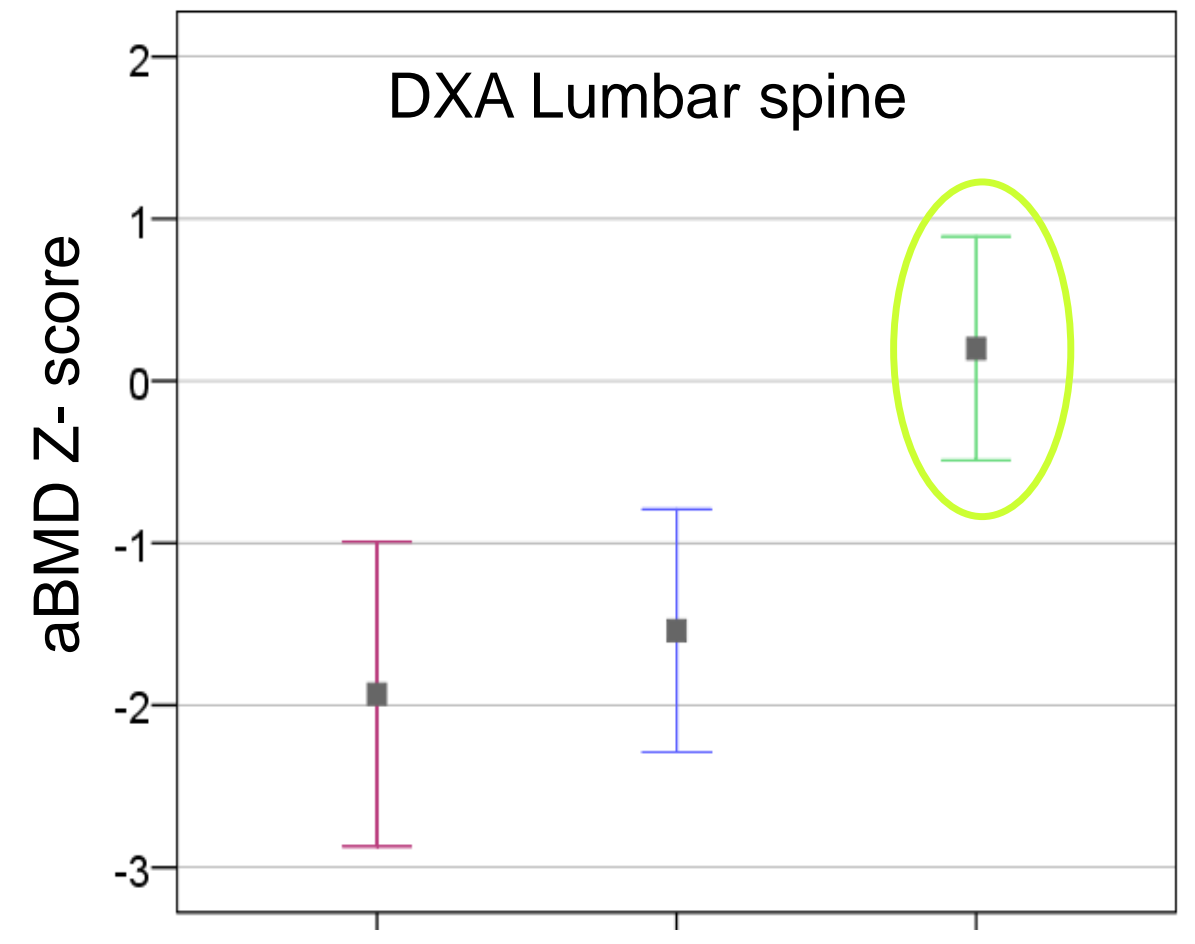

Normal Conductive/ Sensori-

$\begin{array}{lll} & \text { mixed } & \text { neural } \\ (N=19) & (N=29) & (N=8)\end{array}$

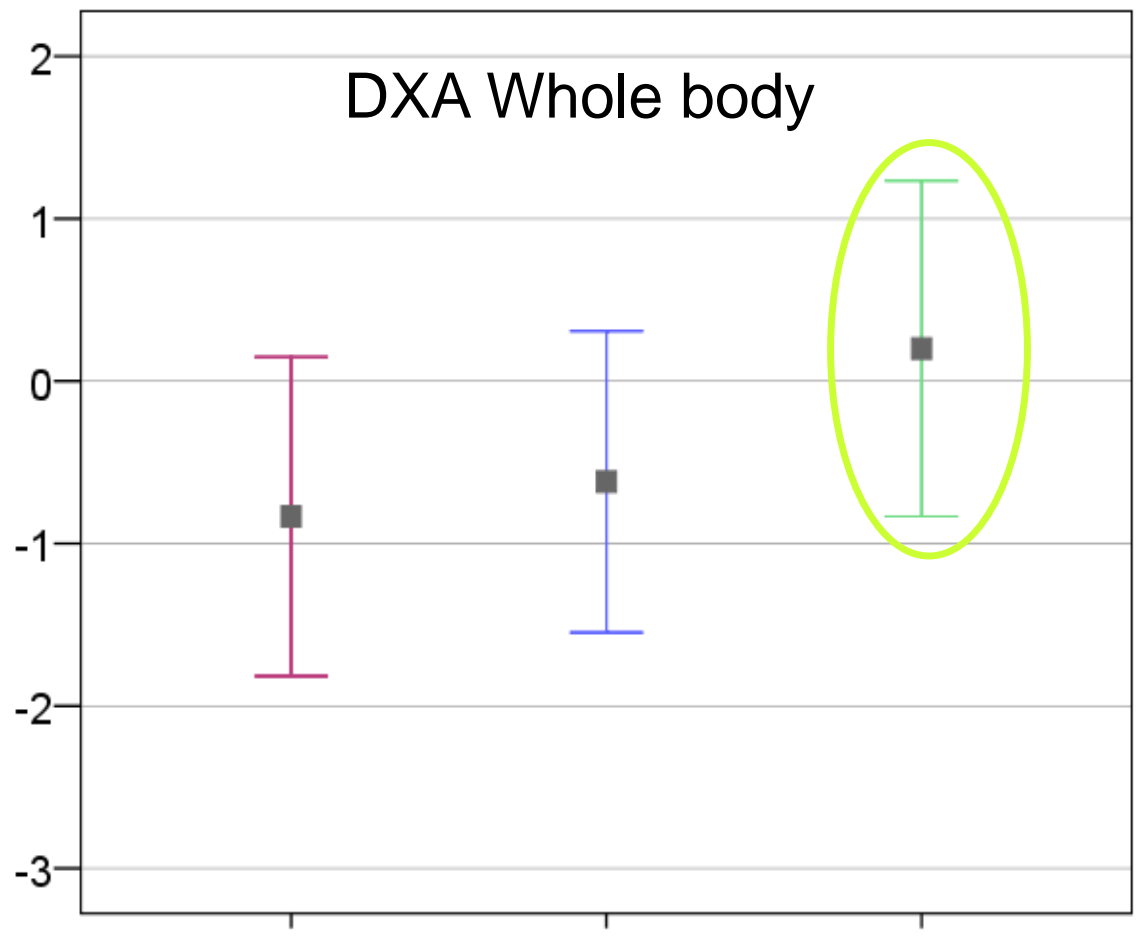

Normal Conductive/ Sensorimixed $(N=19) \quad(N=29)$ neural $(N=8)$

$\square \quad$ Mean z-scores < 0 (except sensorineural losses)

$\square \quad$ ANCOVA $_{\text {[gender, weight, type I collagen defect] : }}$

sensorineural hearing loss $>$ conductive/mixed hearing loss and normal hearing $(P<0.05)$ 


\section{Hearing loss as a function of vBMD (pQCT)}

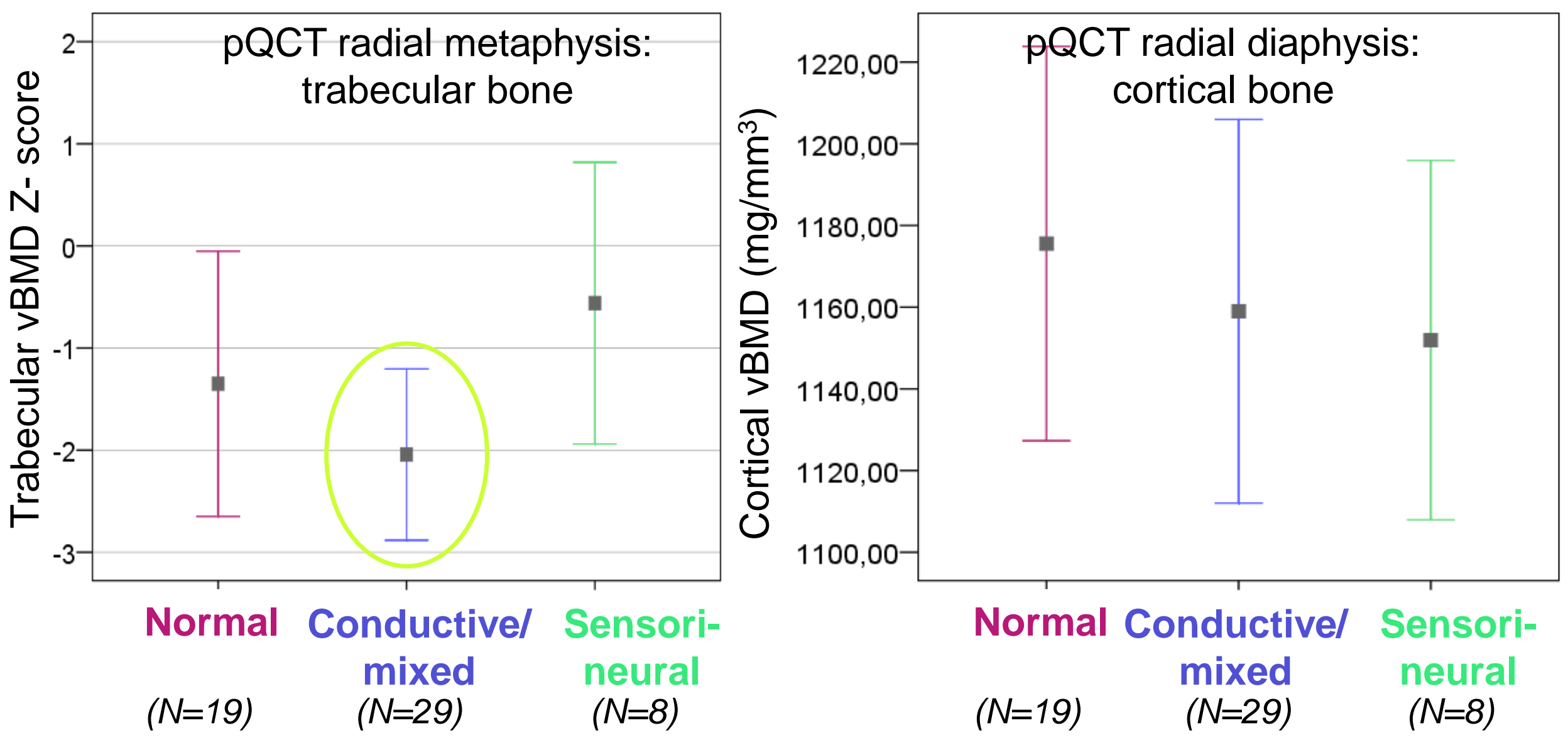

- ANCOVA $_{\text {[gender, age, type I collagen defect] }}$ for (trabecular vBMD z-score *hearing): conductive/mixed hearing loss $<$ normal and sensorineural hearing loss

- Radial diaphysis: no differences in cortical vBMD or bone geometry parameters 


\section{Between-relatives comparisons of BMD and hearing}
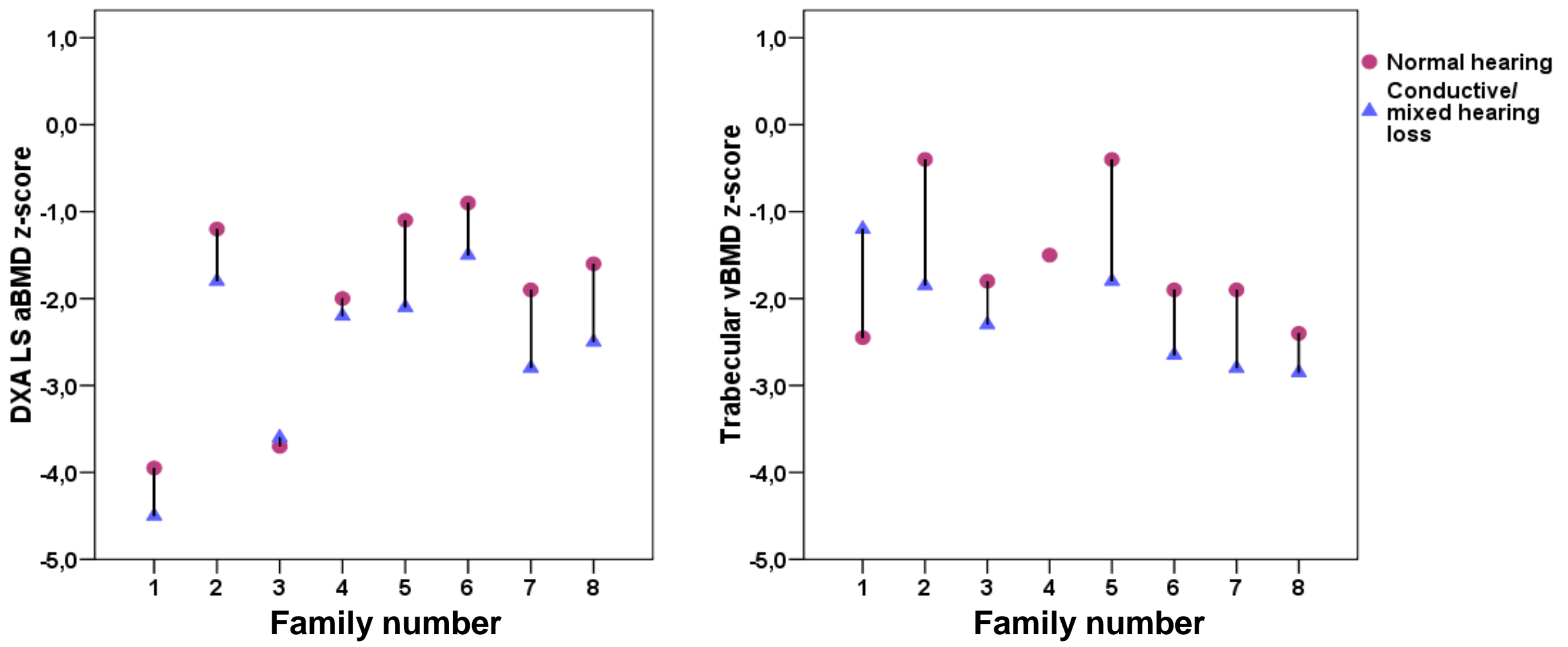

Ol patients with conductive/mixed hearing loss have lower BMD compared to their normal hearing relatives with $\mathrm{Ol}$ 
Ol patients with conductive/mixed hearing loss have lower BMD than patients with normal hearing or pure sensorineural loss

O Ol patients with pure sensorineural hearing loss have higher aBMD than patients with normal hearing or conductive/mixed hearing loss (small sample + highest mean age)

- No differences in volumetric cortical bone mineral density or bone geometry parameters measured at radial diaphysis:

! Cortical vBMD: unreliable parameter when cortical thickness $<2.0 \mathrm{~mm}$ (spatial resolution too low) 


\section{Discussion}

- Temporal bone:

- Cortical bone

- Bone formation complete at age 1 year

- Bone remodeling is minimal

- Association conductive/mixed hearing loss and lower BMD: accumulating microfractures and fatigue microdamage destruct the osteoprotegerin (OPG) pathways which regulate temporal bone remodeling inhibition?

a Future perspectives:

- Replication in large population

- Histological investigations of OI temporal bones

- Effects of bisphosphonates on hearing in OI 
THANK

ias 